\title{
The nuclear force in the third millennium*
}

\begin{abstract}
R. Machleidt
Department of Physics, University of Idaho, Moscow, Idaho 83844, U. S. A.

I will review recent progress in our understanding of the nuclear force. In the course of the 1990's, so-called high-precision, charge-dependent nucleon-nucleon potentials have been constructed which are, essentially, phenomenological models. These potentials are now commonly used as input for exact few-body calculations and microscopic nuclear many-body theory. I will critically analyses those models and point out their strenghts and weaknesses. Particular emphasis will be on charge dependence. Other recent research was conducted on a more basic level: understanding the nuclear force in terms of the fundamental theory of strong interactions, QCD. Predictions from this sector are typically qualitative in nature. Thus, the main problem of the current status in the field is that quantitative models for the nuclear force have a poor theoretical background, while theory based models yield poor results. The chief challenge for the new millenium is to overcome this discrepancy. Chiral effective field theory may be a suitable tool to solve the problem.
\end{abstract}

\section{INTRODUCTION}

The nuclear force is the heart of nuclear physics. Yet, in spite of 70 years of research, we do not fully understand it. I will critically review some recent advances (Sec. 2) and then point out future directions (Sec. 3) that may have the potential to finally crack the case - in the course of the new millennium.

\section{RECENT PROGRESS}

I define 'recent progress' as the progress of the past decade (i. e., the 1990's). In this decade, we have seen advances in $N N$ phenomenology as well as theory. In this section, I will focus on phenomenology. More fundamental issues are addressed in Sec. 3.

\subsection{Phase shift analysis}

In spite of the huge $N N$ database available today, conventional phase shift analyses are by no means perfect. For example, the phase shift solutions obtained by Bugg [1] or the VPI group [2] typically have a $\chi^{2} /$ datum of about 1.4 , for the energy range $0-425 \mathrm{MeV}$. This may be due to inconsistencies in the data as well as deficiencies in the constraints applied in the analysis. In any case, it is a matter of fact that within the conventional phase shifts analysis, in which the lower partial waves are essentially unconstrained, a better fit cannot be achieved.

\footnotetext{
${ }^{*}$ Invited talk presented at the XVIIth European Conference on Few-Body Problems in Physics, Evora, Portugal, September 2000; to be published in Nucl. Phys. A.
} 
About two decades ago, the Nijmegen group embarked on a program to substantially improve $N N$ phase shift analysis. To achieve their goal, the Nijmegen group took two decisive measures [3]. First, they 'pruned' the data base; i.e., they scanned very critically the world $N N$ data base (all data in the energy range between zero and $350 \mathrm{MeV}$ of laboratory energy published in a regular physics journal between January 1955 and December 1992) and eliminated all data that had either an improbably high $\chi^{2}$ (off by more than three standard deviations) or an improbably low $\chi^{2}$; of the 2078 world $p p$ data below $350 \mathrm{MeV}$ 1787 survived the scan, and of the $3446 \mathrm{np}$ data 2514 survived. Secondly, they introduced sophisticated, semi-phenomenological model assumptions into the analysis. Namely, for each of the lower partial waves $(J \leq 4)$ a different energy-dependent potential is adjusted to constrain the energy-dependent analysis. Phase shifts are obtained using these potentials in a Schroedinger equation. From these phase shifts the predictions for the observables are calculated including the $\chi^{2}$ for the fit of the experimental data. This $\chi^{2}$ is then minimized as a function of the parameters of the partial-wave potentials. Thus, strictly speaking, the Nijmegen analysis is a potential analysis; the final phase shifts are the ones predicted by the 'optimized' partial-wave potentials which involve 39 parameters.

In the combined $p p$ and $n p$ analysis [3], the fit for $1787 p p$ data and $2514 n p$ data below $350 \mathrm{MeV}$ results in the 'perfect' $\chi^{2} /$ datum $=0.99$ for the $N N$ database available in 1993. Since then, many $p p$ data of high precision have been taken (particularly at IUCF [4, 5]). Today the $p p$ database consists of 2932 data (below $350 \mathrm{MeV}$ ) for which the Nijmegen analysis produces a $\chi^{2} /$ datum $=1.09$ not quite perfect anymore.

\subsection{The new high-precision $N N$ potentials}

In the 1990's, one focus has been on the quantitative aspect of the $N N$ potentials. Even the best $N N$ models of the 1980's [6,7] fit the $N N$ data typically with a $\chi^{2} /$ datum $\approx 2$ or more. This is still substantially above the perfect $\chi^{2} /$ datum $\approx 1$. To put microscopic nuclear structure theory to a reliable test, one needs a perfect $N N$ potential such that discrepancies in the predictions cannot be blamed on a bad fit of the $N N$ data.

Based upon the Nijmegen analysis and the (pruned) Nijmegen 1992 database, new charge-dependent $N N$ potentials were constructed in the early/mid 1990's. The groups involved and the names of their new creations are, in chronological order:

- Nijmegen group [8]: Nijm-I, Nijm-II, and Reid93 potentials.

- Argonne group [9]: $V_{18}$ potential.

- Bonn group [10,11]: CD-Bonn potential.

All these potentials have in common that they use about 45 parameters and fit the pruned 1992 Nijmegen data base with a $\chi^{2} /$ datum $\approx 1$. However, since 1993 the $p p$ database has substantially expanded and for the current database the $\chi^{2} /$ datum produced by some of these potentials is not so perfect anymore (see Sec. 2.3.2. and Table 2 below).

Concerning the theoretical basis of these potential, one could say that they are allmore or less - constructed 'in the spirit of meson theory' (e.g., all potentials include the one-pion-exchange (OPE) contribution). However, there are considerable differences in the details leading to considerable off-shell differences among the potentials. 
Table 1

Modern high-precision $N N$ potentials and their predictions for the two- and three-nucleon bound state.

\begin{tabular}{|c|c|c|c|c|c|c|}
\hline & CD-Bonn [10,11] & Nijm-I [8] & \begin{tabular}{ll|} 
Nijm-II [8] \\
\end{tabular} & Reid93 [8] & \begin{tabular}{ll|l}
$V_{18}$ & $9]$ \\
\end{tabular} & $\begin{array}{l}\text { NATURE } \\
\end{array}$ \\
\hline Character & nonlocal & mixed $^{a}$ & local & local & local & "nonlocal \\
\hline Deuteron properties: & & & & & & \\
\hline Quadr. moment $\left(\mathrm{fm}^{2}\right)$ & 0.270 & 0.272 & 0.271 & 0.270 & 0.270 & $0.276(3)^{b}$ \\
\hline Asymptotic D/S state & 0.0256 & 0.0253 & 0.0252 & 0.0251 & 0.0250 & $0.0256(4)$ \\
\hline D-state probab. (\%) & 4.85 & 5.66 & 5.64 & 5.70 & 5.76 & - \\
\hline $\begin{array}{l}\text { Triton binding }(\mathrm{MeV}) \text { : } \\
\text { nonrel. calculation }\end{array}$ & 8.00 & 7.72 & 7.62 & 7.63 & 7.62 & - \\
\hline relativ. calculation & 8.2 & - & - & - & - & 8.48 \\
\hline
\end{tabular}

${ }^{a}$ Central force nonlocal, tensor force local.

${ }^{b}$ Corrected for meson-exchange currents and relativity.

The CD-Bonn potential uses the full, original, nonlocal Feynman amplitude for OPE, while all other potentials apply local approximations. As a consequence of this, the CDBonn potential has a weaker tensor force as compared to all other potentials. This is reflected in the predicted D-state probabilities of the deuteron, $P_{D}$, which is a measure of the strength of the nuclear tensor force. While CD-Bonn predicts $P_{D}=4.85 \%$, the other potentials yield $P_{D}=5.7(1) \%$ (cf. Table 1). These differences in the strength of the tensor force lead to considerable differences in nuclear structure predictions. An indication of this is given in Table 1: The CD-Bonn potentials predicts $8.00 \mathrm{MeV}$ for the triton binding energy, while the local potentials predict only $7.62 \mathrm{MeV}$. More discussion of this aspect can be found in Ref. 12,13.

The OPE contribution to the nuclear force essentially takes care of the long-range interaction and the tensor force. In addition to this, all models must describe the intermediate and short range interaction, for which very different approaches are taken. The CD-Bonn includes (besides the pion) the vector mesons $\rho(769)$ and $\omega(783)$, and two scalar-isoscalar bosons, $\sigma$, using the full, nonlocal Feynman amplitudes for their exchanges. Thus, all components of the CD-Bonn are nonlocal and the off-shell behavior is the original one as determined from relativistic field theory.

The models Nijm-I and Nijm-II are based upon the Nijmegen78 potential [14] which is constructed from approximate one-boson-exchange (OBE) amplitudes. Whereas NijmII uses the totally local approximations for all OBE contributions, Nijm-I keeps some nonlocal terms in the central force component (but the Nijm-I tensor force is totally local). However, nonlocalities in the central force have only a very moderate impact on nuclear structure. Thus, it would be more important to keep the tensor force nonlocalities.

The Reid93 [8] and Argonne $V_{18}$ [9] potentials do not use meson-exchange for intermediate and short range; instead, a phenomenological parametrization is chosen. The Argonne $V_{18}$ uses local functions of Woods-Saxon type, while Reid93 applies local Yukawa functions of multiples of the pion mass, similar to the original Reid potential of 1968 [15]. At very short distances, the potentials are regularized either by exponential ( $V_{18}$, Nijm-I, Nijm-II) or by dipole (Reid93) form factors, which are all local functions. 


\subsection{How perfect are the 'perfect potentials'?}

By now, the new high-precision $N N$ potentials have been around for a while. It is therefore appropriate to take a second look at them. To properly discuss the above question, we need to distinguish between the theoretical and practical point of view. On theoretical grounds, the new potentials are not perfect at all. In fact, they are a step backwards. Already in the 1980's, we had potentials [6,7] with a better theoretical basis than any of the potentials of the 1990's. However, one has to concede that the new high-precision potentials were developed essentially for practical reasons. One wants to use them as reliable input for exact few-body calculations and other microscopic nuclear structure problems. Therefore, we should judge these potentials mainly in regard to their quantitative nature. The question then is: Are these potentials perfect, at least, on quantitative grounds? We will discuss now some crucial quantitative aspects.

\subsubsection{Charge dependence}

By definition, charge independence is invariance under any rotation in isospin space. A violation of this symmetry is referred to as charge dependence or charge independence breaking (CIB). Charge symmetry is invariance under a rotation by $180^{\circ}$ about the $y$ axis in isospin space if the positive $z$-direction is associated with the positive charge. The violation of this symmetry is known as charge symmetry breaking (CSB). Obviously, CSB is a special case of charge dependence.

CIB of the strong $N N$ interaction means that, in the isospin $T=1$ state, the protonproton $\left(T_{z}=+1\right)$, neutron-proton $\left(T_{z}=0\right)$, or neutron-neutron $\left(T_{z}=-1\right)$ interactions are (slightly) different, after electromagnetic effects have been removed. CSB of the $N N$ interaction refers to a difference between proton-proton $(p p)$ and neutron-neutron ( $n n$ ) interactions, only. For recent reviews, see Refs. 16,17.

All new potentials are charge-dependent since this is essential for obtaining a good $\chi^{2}$ for the fit of the $p p$ as well as $n p$ data. Thus, each potential comes in three variants: $p p$, $n p$, and $n n$.

\section{Charge symmetry breaking (CSB)}

The difference between the masses of neutron and proton represents the most basic cause for CSB of the nuclear force. Therefore, it is important to have a very thorough accounting of this effect. The most trivial consequence of nucleon mass splitting is a difference in the kinetic energies: for the heavier neutrons, the kinetic energy is smaller than for protons. This raises the magnitude of the $n n$ scattering length by $0.3 \mathrm{fm}$ as compare to $p p$. The nucleon mass difference also affects the one-boson-exchange (OBE) contributions to the $N N$ potential, but only by a negligible amount. In summary, the two most obvious and trivial CSB effects explain only about $20 \%$ of the empirical CSB splitting of the ${ }^{1} S_{0}$ scattering lenght which is $\Delta a_{C S B}=1.6 \pm 0.6 \mathrm{fm}$ [17 19$]$.

Some models for the nuclear force (e. g., the Nijmegen potentials [8]) include only the two CSB effects just dicussed and, thus, leave CSB essentially unexplained. Or, in other words, the Nijmegen group does not offer any genuine neutron-neutron potentials. In some other models (e. g., the Argonne $V_{18}$ potential [9]), a purely phenomenological term is added to the potential that fits $\Delta a_{C S B}$.

The point we like to make here is that one can do much better than this in dealing with 


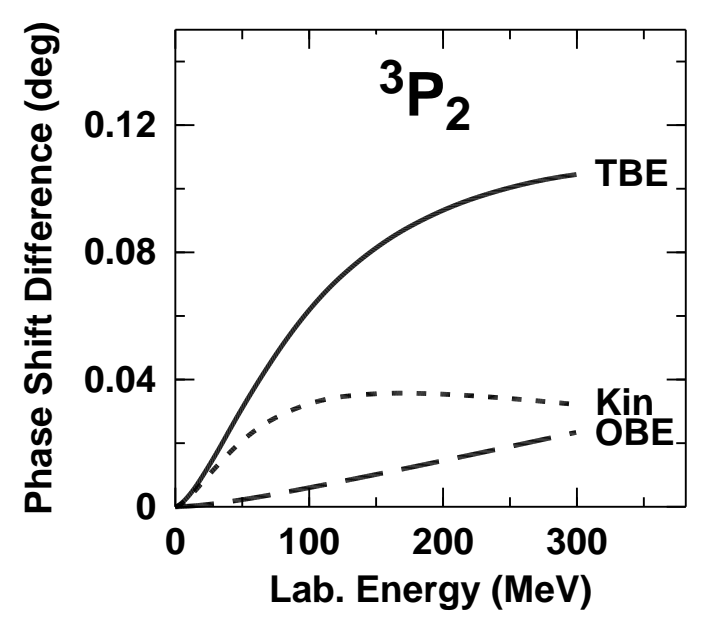

Figure 1. CSB phase shift differences in the ${ }^{3} P_{2}$ state due to the impact of nucleon mass splitting on kinematics (dotted line labeled 'Kin'), one-boson exchange (dashed, OBE), and two-boson exchange diagrams (solid, TBE).

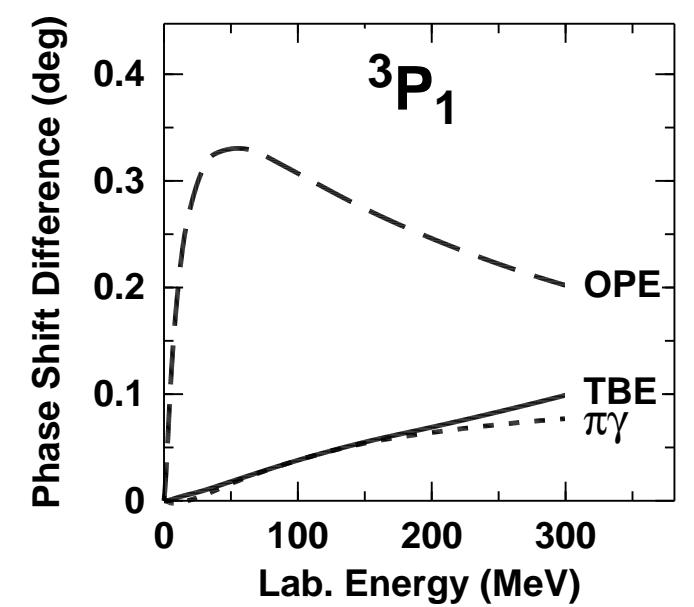

Figure 2. CIB phase shift differences in the ${ }^{3} P_{1}$ state due to the effect from pion mass splitting on OPE (dashed line) and two-boson exchange diagrams (solid, TBE). CIB from irreducible $\pi \gamma$ exchange is shown by the dotted curve.

the CSB of the nuclear force. In Ref. [20], the CSB effect due to nucleon mass splitting from irreducible diagrams of two-boson exchange (TBE) was calculated thoroughly for all two-nucleon partial waves with $J \leq 4$. It was found that this effect is relatively large and fully explains the empirical CSB splitting of the singlet scattering length. The major part of the CSB effect comes from diagrams of $2 \pi$ exchange where those with $N \Delta$ intermediate states make the largest contribution [21. Noticeable CSB effects occur also in $P$ (cf. Fig. 1) and $D$ waves.

Because of the outstanding importance of the CSB effect from TBE, it should be included in $N N$ force models (and, therefore, it has been incorporated in the latest update of the CD-Bonn potential [11]). To have distinct $p p$ and $n n$ potentials is important for addressing several interesting issues in nuclear physics, like the ${ }^{3} \mathrm{H}-{ }^{3} \mathrm{He}$ binding energy difference for which the CD-Bonn potential predicts $60 \mathrm{keV}$ in agreement with empirical estimates. Another issue is the Nolen-Schiffer (NS) anomaly 22 regarding the energies of neighboring mirror nuclei. Potentials that do not include any CSB have no chance to ever explain this phenomenon. Some potentials that include CSB focus on the ${ }^{1} S_{0}$ state only, since this is where the most reliable empirical information is. However, even this is not good enough. A recent study 23] has shown that the CSB in partial waves with $L>0$ as derived from the Bonn model is crucial for a quantitative explanation of the NS anomaly.

\section{Charge independence breaking (CIB)}

The major cause of CIB in the $N N$ interaction is pion mass splitting. Based upon the Bonn Full Model for the $N N$ interaction [7], the CIB due to pion mass splitting has been 
calculated carefully and systematically in Ref. [24]. The largest CIB effect comes from one-pion-exchange (OPE) which accounts for about $50 \%$ of the empirical CIB splitting of the ${ }^{1} S_{0}$ scattering length, $\Delta a_{C I B}=5.7 \pm 0.3 \mathrm{fm}$ [17]. Due to the small mass of the pion, OPE is also a sizable contribution in all partial waves with $L>0$; and due to the pion's relatively large mass splitting (3.4\%), OPE creates relatively large chargedependent effects in all partial waves. Therefore, all modern phase shift analyses [2,3] and all modern $N N$ potentials 8 11] include the CIB effect created by OPE.

However, pion mass splitting creates further CIB effects through the diagrams of $2 \pi$ exchange and other two-boson exchange diagrams that involve pions. The evaluation of this CIB contribution is very involved, but it has been accomplished in Ref. [24]. The CIB effect from all the relevant two-boson exchanges (TBE) contributes about $1.3 \mathrm{fm}$ to $\Delta a_{C I B}$. Concerning phase shift differences, it is noticeable up to $D$ waves and can amount up to $50 \%$ of the OPE effect in some states (see Fig. 2).

Another source of CIB is irreducible $\pi \gamma$ exchange. Recently, these contributions have been evaluated in the framework of chiral perturbation theory by van Kolck et al. [25]. Based upon this work, we have calculated the impact of the $\pi \gamma$ diagrams on the ${ }^{1} S_{0}$ scattering length and on $n p$ phase shifts. In $L>0$ states, the size of this contribution is typically the same as the CIB effect from TBE.

From Fig. 2 it is evident that TBE and $\pi \gamma$ create sizable CIB effects in states with $L>0$. Therefore, a thoroughly constructed, modern, charge-dependent $N N$ potential should include them. The $N N$ potentials [8,9] ignore these contributions while the latest CD-Bonn update [11] incorporates them.

\subsubsection{After-1992 $N N$ data}

After 1992, there has been a fundamental breakthrough in the development of experimental methods for conducting hadron-hadron scattering experiments. In particular, the method of internal polarized gas targets applied in stored, cooled beams is now working perfectly in several hadron facilities, e. g., IUCF and COSY. Using this new technology, IUCF has produced a large number of $p p$ spin correlation parameters of very high precision. The new IUCF data [4, (5] (together with a few other recent $p p$ data) amount to 1145 pieces of new $p p$ data (below $350 \mathrm{MeV}$ ). This should be compared to the number of $p p$ data of the pre-1993 era, namely, 1787. Thus, the $p p$ database has increased by about $2 / 3$ since 1993. The importance of the new $p p$ data is further enhanced by the fact that they are of much higher quality than the old ones. Therefore, the after-1992 $p p$ data represent a true challenge for all phase shift analyses and high-quality $N N$ potentials.

In Table 2 we show results. The three databases used in this table are defined as follows. The 1992 database (or pre-1993 database) is identical to the one used by the Nijmegen group for their phase shift analysis [3]. It consists of all $N N$ data below 350 $\mathrm{MeV}$ that were published between January 1955 and December 1992 (and not rejected in the Nijmegen data analysis). The after-1992 database includes all $N N$ data (below 350 $\mathrm{MeV}$ ) published between January 1993 and December 1999. Finally, the 1999 database is the sum of the 1992 base and the after-1992 data and, thus, represents the world $N N$ data below $350 \mathrm{MeV}$ available in the year of 2000 .

What stands out in Table 2 are the rather large values for the $\chi^{2} /$ datum generated by the Nijmegen analysis and the Argonne potential for the the after-1992 $p p$ data, which 
Table 2

$\chi^{2} /$ datum for the reproduction of the $p p$ data by the Nijmegen phase shift analysis [3], the Argonne $V_{18}$ potential [9], and the CD-Bonn potential [11]. The Nijmegen potentials (Nijm-I, Nijm-II, Reid93) [8] produce $\chi^{2}$ similar to the Nijmegen PSA.

\begin{tabular}{lccc}
\hline & Nijmegen & Argonne & CD-Bonn \\
& phase shift analysis & $V_{18}$ potential & potential \\
\hline $1992 p p$ database $($ (1787 data) & 1.00 & 1.10 & 1.00 \\
After-1992 $p p$ data (1145 data) & $\mathbf{1 . 2 4}$ & $\mathbf{1 . 7 4}$ & $\mathbf{1 . 0 3}$ \\
$1999 p p$ database $(2932$ data) & 1.09 & 1.35 & 1.01 \\
\hline
\end{tabular}

are essentially the new IUCF $p p$ spin correlation parameters [4] and spin transfer coefficients [5]. The Nijmegen potentials [8] produce $\chi^{2}$ that are very similar to the ones by the Nijmegen analysis. Clearly, for Nijmegen and Argonne, the $\chi^{2} /$ datum is not perfect anymore. This fact is a clear indication that these new data provide a very critical test/constraint for any $N N$ model. It further indicates that fitting the pre-1993 $p p$ data does not nessarily imply a good fit of those IUCF data. On the other hand, fitting the new IUCF data does imply a good fit of the pre-1993 data. The conclusion from these two facts is that the new IUCF data provide information that was not contained in the old database. Or, in other words, the pre-1993 data were insufficient and still left too much lattitude for pinning down $N N$ models. One thing in particular that we noticed is that the ${ }^{3} P_{1}$ phase shifts above $100 \mathrm{MeV}$ have to be lower than the values given in the Nijmegen analysis.

\subsubsection{Extrapolating low-energy potentials to higher energies}

$N N$ potentials designed for nuclear structure purposes are typically fitted to the $N N$ scattering data up to pion production thereshold or slightly beyond (e. g., $350 \mathrm{MeV}$ ). A very basic reason for this is that a real potential cannot describe the inelasticities of particle production. On the other hand, nuclear structure calculations are probably sensitive to the properties of a potential above $350 \mathrm{MeV}$. For example, the Brueckner $G$ matrix, which is a crucial quantity in many microscopic approaches to nuclear structure, is the solution of the integral equation,

$G\left(\mathbf{q}^{\prime}, \mathbf{q}\right)=V\left(\mathbf{q}^{\prime}, \mathbf{q}\right)-\int d^{3} k V\left(\mathbf{q}^{\prime}, \mathbf{k}\right) \frac{M^{\star} Q}{k^{2}-q^{2}} G(\mathbf{k}, \mathbf{q})$

Notice that the potential $V$ is involved in this equation for all momenta from zero to infinity, on- and off-shell. Now, it may very well be true that, as the momenta increase, their importance may decrease (due to the short-range repulsion of the nuclear force and the associated short-range suppression of the nuclear wave function). However, it is also true that the impact of the potential does not suddenly drop to zero as soon as the momenta involved become larger than the equivalent of $350 \mathrm{MeV}$ lab. energy. Thus, there are good physics arguments why $N N$ potentials should extrapolate in a reasonable way towards higher energies.

We have investigated this issue and found good and bad news. The good news is that most potentials reproduce in most partial waves the $N N$ phase shifts up to about $1000 \mathrm{MeV}$ amazingly well. The bad news is that there are some singular cases where 

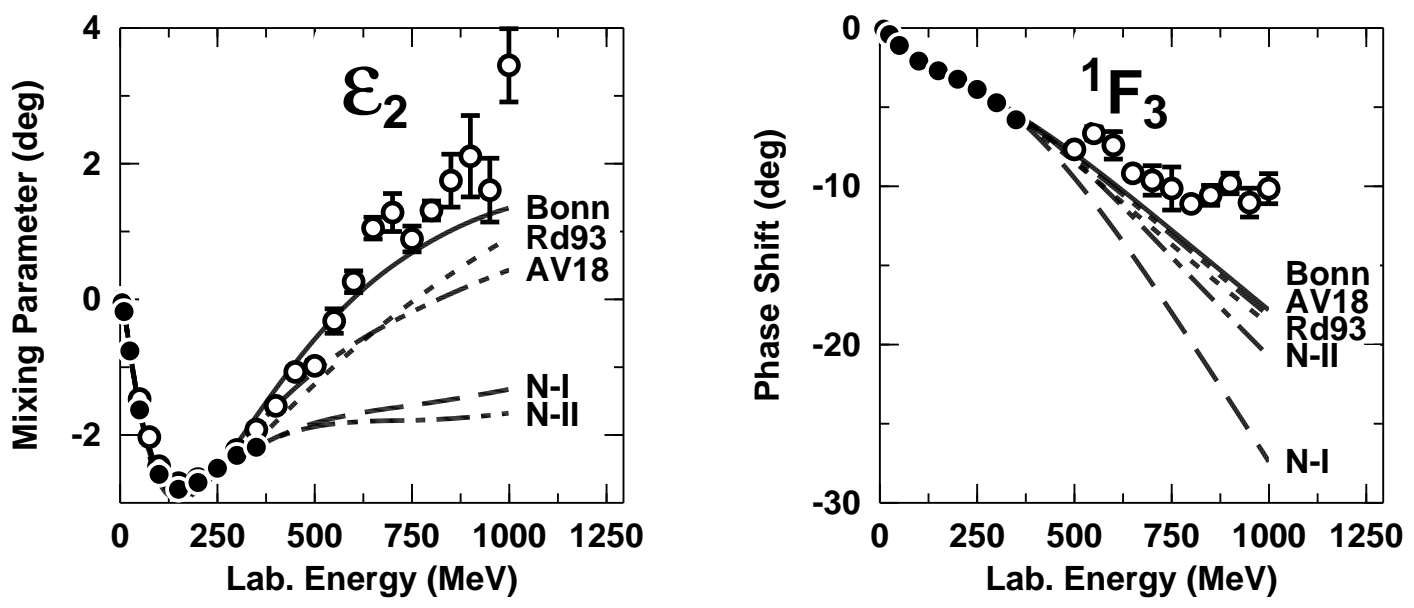

Figure 3. The $\epsilon_{2}$ mixing parameter and the ${ }^{1} F_{3}$ phase shift up to $1000 \mathrm{MeV}$ lab. energy for various potentials as denoted (N-I and N-II refer to the Nijmegen potentials). Solid dots represent the Nijmegen PSA [3] and open circles the VPI analysis SM99 [2].

the reproduction of phase parameters for higher energies is disturbingly bad. The two most notorious cases are shown in Fig. 3. Above $350 \mathrm{MeV}$, the $\epsilon_{2}$ mixing parameter is substantially underpredicted by both Nijmegen potentials (N-I and N-II). The reason for this is that, for $\epsilon_{2}$, both potentials follow very closely the Nijmegen PSA [3] (solid dots in Fig. 3) up to $350 \mathrm{MeV}$. Thus, these potentials are faithfull extrapolations of the Nijmegen PSA to higher energies. Since this extrapolation is wrong, the suspicion is that the Nijmegen PSA has a wrong trend in the energy range 250-350 MeV. New data on $p p$ spin transfer coefficients [5] in the energy range 300-500 MeV could resolve the issue.

A similar problem occurs in ${ }^{1} F_{3}$ (Fig. 3). Here, the dashed curve (N-I) is the extrapolation of the Nijmegen PSA, indicating that the analysis may have the wrong trend in the energy range $200-350 \mathrm{MeV}$.

We note that, in the two channels discussed, the inelasticity has little impact on the phase parameters shown and would not fix the problems.

The moral is that one should not follow just one analysis, particularly, if that analysis is severely limited in its energy range. It is important to also keep the broad picture in mind.

\subsubsection{Summary}

We asked the question: How perfect are the perfect potentials - on quantitative grounds? The concise and discreet answer is: Some high-quality potentials have room for improvement.

\section{FUTURE DIRECTIONS}

\subsection{Critical summary of current status}

During the past decade or so, the research on the $N N$ interaction has proceeded essentially along two lines. There was the phenomenological line which has produced the 
high-precision, charge-dependent $N N$ potentials 811. This was practical work, necessary to provide reliable input for exact few-body calculations and nuclear many-body theory.

The goal of the second line of research was to approach the problem on a more fundamental level. Since about 1980, we have seen many efforts to derive the nuclear force from the underlying theory of strong interactions, quantum chromodynamics (QCD). Due to its nonperturbative character in the low-energy regime, QCD cannot be solved exactly for the problem under consideration. Therefore, so-called QCD-related or QCD-inspired models have been developed, like, Skyrmion or Soliton models [26] and constituent quark cluster models 27,28]. The success of these efforts is mixed. Predictions are typically only of qualitative nature; and if predictions are quantitative then it's due to an admixture of traditional meson exchanges.

In summary, one problem of the current status in the field is that quantitative models for the nuclear force have only a poor theoretical background, while theory based models yield only poor results. This discrepancy between theory and practice has become rather larger than smaller, in the course of the 1990s. Another problem is that the 'theory based models' are not strictly derived from QCD, they are modeled after QCD - often with handwoven arguments. Thus, one may argue that these models are not any better than the traditional meson-exchange models (that are nowadays perceived as phenomenology). The purpose of physics is to explain nature in fundamental terms. The two trends just discussed are moving us away from this aim, which is reason for serious concern.

Therefore, the main goal of future research on the nuclear force must be to overcome the above discrepancies. To achieve this goal, we need a basic theory that is amenable to calculation and yields quantitative results.

\subsection{The Effective Field Theory Concept}

In recent years, the concept of effective field theories (EFT) has drawn considerable attention in particle and nuclear physics [29 31]. The notion of effective field theories may suggest a difference to fundamental field theories. However, it is quite likely that all field theories (including those that we perceive presently as fundamental) are effective in the sense that they are low-energy approximations to some 'higher' theory.

The basis of the EFT concept is the recognition of different energy scales in nature. Each energy level has its characteristic degrees of freedom. As the energy increases and smaller distances are probed, new degrees of freedom become relevant and must be included. Conversely, when the energy drops, some degrees of freedom become irrelevant and are frozen out.

To model the low-energy theory, one relies on a famous 'folk theorem' by Weinberg [32, [33] which states:

If one writes down the most general possible Langrangian, including all terms consistent with assumed symmetry principles, and then calculates matrix elements with this Langrangian to any given order of perturbation theory, the result will simply be the most general possible S-matrix consistent with analyticity, perturbative unitarity, cluster decomposition, and the assumed symmetry principles.

The essential point of an effective field theory is that we are not allowed to make any 
assumption of simplicity about the Lagrangian and, consequently, we are not allowed to assume renormalizability. The Langrangian must include all possible terms, because this completeness guarantees that the effective theory is indeed the low-energy limit of the underlying theory. Now, this implies that we are faced with an infinite set of interactions. To make the theory managable, we need to organize a perturbation expansion. Then, up to a certain order in this expansion, the number of terms that contribute is finite and the theory will yield a well-defined result.

In strong interactions, the transition from the 'fundamental' to the effective level happens through a phase transition that takes place around $\Lambda_{Q C D} \approx 1 \mathrm{GeV}$ via the spontaneous breaking of chiral symmetry which generates pseudoscalar Goldstone bosons. Therefore, at low energies $\left(E<\Lambda_{Q C D}\right)$, the relevant degrees of freedom are not quarks and gluons, but pseudoscalar mesons and other hadrons. Approximate chiral symmetry is reflected in the smallness of the masses of the pseudoscalar mesons. The effective theory that describes this scenario is known as chiral perturbation theory $(\chi \mathrm{PT})$ 30, 34, 35.

If we believe in the basic ideas of EFT, then, at low energies, $\chi \mathrm{PT}$ is as fundamental as QCD at high energies. Moreover, due to its perturbative arrangement, $\chi \mathrm{PT}$ can be calculated: order by order. So, here we may have what we were asking for at the end of the previous sub-section: a basic theory that is amenable to calculation. Therefore, $\chi \mathrm{PT}$ has the potential to overcome the discrepancy between theory and practice that has beset the theoretical research on the nuclear force for so many years.

\subsection{Chiral perturbation theory and nuclear forces}

The idea to derive nuclear forces from chiral effective Lagrangians is not new. A program was started some 10 years ago by Weinberg [36,37], Ordóñez [38], and van Kolck [39 42].

After the program was initiated, considerable activity ensued [43]. Even though all authors start from chiral effective Langrangians, there are considerable differences in the details. Among all these effeorts, the recent work by Epelbaum, Glöckle, and Meißner 44446] is particularly promising. The details of how to derive the $N N$ potential from chiral effective Langrangians (up to next-to-next-to-leading order) are explained in the contribution by Epelbaum to these proceedings [46]. Therefore, I will not go into this and, instead, recommend to the interested reader to study this beautiful contribution.

However, I like to point out one attractive point of the $\chi \mathrm{PT}$ approach. If, in the traditional approach, one wants to reproduce, e. g., the experimental binding energies of the triton, the alpha particle or other nuclei, one complements the $N N$ potential with a (phenomenological) three-nucleon force (3NF) [47]. Since different $N N$ potentials leave different discrepancies to experiment (cf. Table 1), the $3 \mathrm{NF}$ is adjusted from potential to potential. From a more fundamental point of view, this proceedure is very unsatisfactory, since it lacks any underlying systematics. However, within the framework of traditional meson theory, there is nothing else you can do, because there is no a priori connection between the off-shell $N N$ potential and the existence of certain many-body forces.

In the framework of $\chi \mathrm{PT}$, there is this connection from the outset. In each order of $\chi \mathrm{PT}$, the two-nucleon force is well-defined on- and off-shell and it is also well-defined which $3 \mathrm{NF}$ terms occur in that order. At least that's how it should work 'in theory'. How it works out in practise remains to be seen. We need very accurate chiral potentials to pin down these subtleties. 


\section{CONCLUSIONS}

In the past decade, we had essentially two major advances. On the practical side, high-precision phenomenological $N N$ potentials were constructed (some of which need improvement to keep up with new, more accurate $N N$ data and recent progress in chargedependence). On the theoretical side, nuclear physicists became conscious of chiral symmetry (an important symmetry of QCD). The dynamics created by this symmetry may finally produce $N N$ potentials that are theoretically sound and of 'high-precision'.

This work was supported in part by the US National Science Foundation under GrantNo. PHY-9603097.

\section{REFERENCES}

1. D. V. Bugg and R. A. Bryan, Nucl. Phys. A540, 449 (1992).

2. R. A. Arndt, I. I. Strakovsky, and R. L. Workman, Phys. Rev. C 50, 2731 (1994); ibid. 52, 2246 (1995); see also: SAID, Scattering Analysis Interactive Dial-in program, VPI and GWU.

3. V. G. J. Stoks, R. A. M. Klomp, M. C. M. Rentmeester, and J. J. de Swart, Phys. Rev. C 48, 792 (1993).

4. W. Haeberli et al., Phys. Rev. C 55, 597 (1997); F. Rathmann et al., Phys. Rev. C 58, 658 (1998); B. v. Przewoski et al., Phys. Rev. C 58, 1897 (1998); B. Lorentz et al., Phys. Rev. C 61, 054002 (2000).

5. S. W. Wissink et al., Phys. Rev. Lett. 83, 4498 (1999).

6. M. Lacombe, B. Loiseau, J. M. Richard, R. Vinh Mau, J. Côté, P. Pirès, and R. de Tourreil, Phys. Rev. C 21, 861

7. R. Machleidt, K. Holinde, and Ch. Elster, Phys. Rep. 149, 1 (1987).

8. V. G. J. Stoks, R. A. M. Klomp, C. P. F. Terheggen, and J. J. de Swart, Phys. Rev. C 49, 2950 (1994).

9. R. B. Wiringa, V. G. J. Stoks, and R. Schiavilla, Phys. Rev. C 51, 38 (1995).

10. R. Machleidt, F. Sammarruca, and Y. Song, Phys. Rev. C 53, R1483 (1996).

11. R. Machleidt, The high-precision, charge-dependent, Bonn nucleon-nucleon potential (CD-Bonn), arXiv:nucl-th/0006014.

12. R. Machleidt, Adv. Nucl. Phys. 19, 189 (1989).

13. R. Machleidt, Nuclear Forces and Nuclear Structure, Proc. Nuclear Structure 98, Gatlinburg, Tennessee, 1998, AIP Conf. Proc. 481, edited by C. Baktash (AIP, Woodbury, N.Y., 1999) p. 3.

14. M. M. Nagels, T. A. Rijken, and J. J. de Swart, Phys. Rev. D 17, 768 (1978).

15. R. V. Reid, Ann. Phys. (N.Y.) 50, 411 (1968).

16. G. A. Miller and W. H. T. van Oers, In Symmetries and Fundamental Interactions in Nuclei, W. C. Haxton and E. M. Henley, eds. (World Scientific, Singapore, 1995) p. 127.

17. G. A. Miller, M. K. Nefkens, and I. Slaus, Phys. Rep. 194, 1 (1990).

18. C. R. Howell et al., Phys. Lett. B444, 252 (1998).

19. D. E. González Trotter et al., Phys. Rev. Lett. 83, 3788 (1999).

20. G. Q. Li and R. Machleidt, Phys. Rev. C 58, 1393 (1998).

21. S. A. Coon and J. A. Niskanen, Phys. Rev. C 53, 1154 (1996). 
22. J. A. Nolen and J. P. Schiffer, Annu. Rev. Nucl. Sci. 19, 471 (1969).

23. H. Müther, A. Polls, and R. Machleidt, Phys. Lett. B445, 259 (1999).

24. G. Q. Li and R. Machleidt, Phys. Rev. C 58, 3153 (1998).

25. U. van Kolck, M. C. M. Rentmeester, J. L. Friar, T. Goldman, and J. J. de Swart, Phys. Rev. Lett. 80, 4386 (1998).

26. A. Jackson, A. D. Jackson, and V. Pasquier, Nucl. Phys. A432, 567 (1985); R. Vinh Mau, M. Lacombe, B. Loiseau, W. N. Cottingham, and P. Lisboa, Phys. Lett. 150B, 259 (1985); T. S. Walhout and J. Wambach, Int. J. Mod. Phys. E1, 665 (1992); S. Pepin, Fl. Stancu, W. Koepf, and L. Wilets, Phys. Rev. C 53, 1368 (1996);

27. K. Maltman and N. Isgur, Phys. Rev. D 29, 952 (1984); F. Myhrer and J. Wroldsen, Rev. Mod. Phys. 60, 629 (1988); S. Takeuchi, K. Shimizu, and K. Yazaki, Nucl. Phys. A504, 777 (1989); F. Fernandez, A. Valcarce, U. Straub, and A. Faessler, J. Phys. G: Nucl. Part. Phys. 19, 2013 (1993); D. R. Entem, F. Fernandez, and A. Valcarce, Phys. Rev. C 62, 034002 (2000); K. Shimizu and L. Ya. Glozman, Phys. Lett. B477, $59(2000)$.

28. F. Fernandez, contribution to these proceedings.

29. H. Georgi, Annu. Rev. Nucl. Part. Sci. 43, 209 (1993).

30. G. Ecker, Prog. Part. Nucl. Phys. 35, 1 (1995).

31. D. B. Kaplan, Effective Field Theories, arXiv:nucl-th/9506035.

32. S. Weinberg, Physica 96A, 327 (1979).

33. S. Weinberg, What is Quantum Field Theory, and What Did We Think It Is?, Talk presented at the conference "Historical Examination and Philosophical Reflections on the Foundations of Quantum Field Theory", Boston University, March 1996; in: Boston 1996, Conceptual foundations and quantum field theory, p. 241; arXiv:hep-ph/9702027.

34. H. Leutwyler, Ann. Phys. (N.Y.) 235, 165 (1994).

35. V. Bernard, N. Kaiser, and U.-G. Meißner, Int. J. Mod. Phys. E 4, 193 (1995).

36. S. Weinberg, Phys. Lett. B 251, 288 (1990); Nucl. Phys. B363, 3 (1991).

37. S. Weinberg, Phys. Lett. B 295, 114 (1992).

38. C. Ordonez and U. van Kolck, Phys. Lett. B 291, 459 (1992).

39. U. van Kolck, Ph.D. thesis, University of Texas, 1993; University of Washington Report DOE/ER/40427-13-N94.

40. C. Ordonez, L. Ray, and U. van Kolck, Phys. Rev. Lett. 72, 1982 (1994); Phys. Rev. C 53, 2086 (1996).

41. U. van Kolck, Phys. Rev. C 49, 2932 (1994).

42. U. van Kolck, Prog. Part. Nucl. Phys. 43, 337 (1999).

43. For a recent review that includes a comprehensive list of references, see:

S. R. Beane, P. F. Bedaque, W. C. Haxton, D. R. Phillips, and M. J. Savage, arXiv:nucl-th/0008064.

44. E. Epelbaoum, W. Glöckle, and U.-G. Meißner, Nucl. Phys. A637, 107 (1998).

45. E. Epelbaum, W. Glöckle, and U.-G. Meißner, Nucl. Phys. A671, 295 (2000).

46. E. Epelbaum, contribution to these proceedings.

47. A. Nogga, H. Kamada, and W. Glöckle, Phys. Rev. Lett. 85, 944 (2000). 